\title{
Giant liver cyst caused by tuberculosis infection and masquerading as malignant peritonitis
}
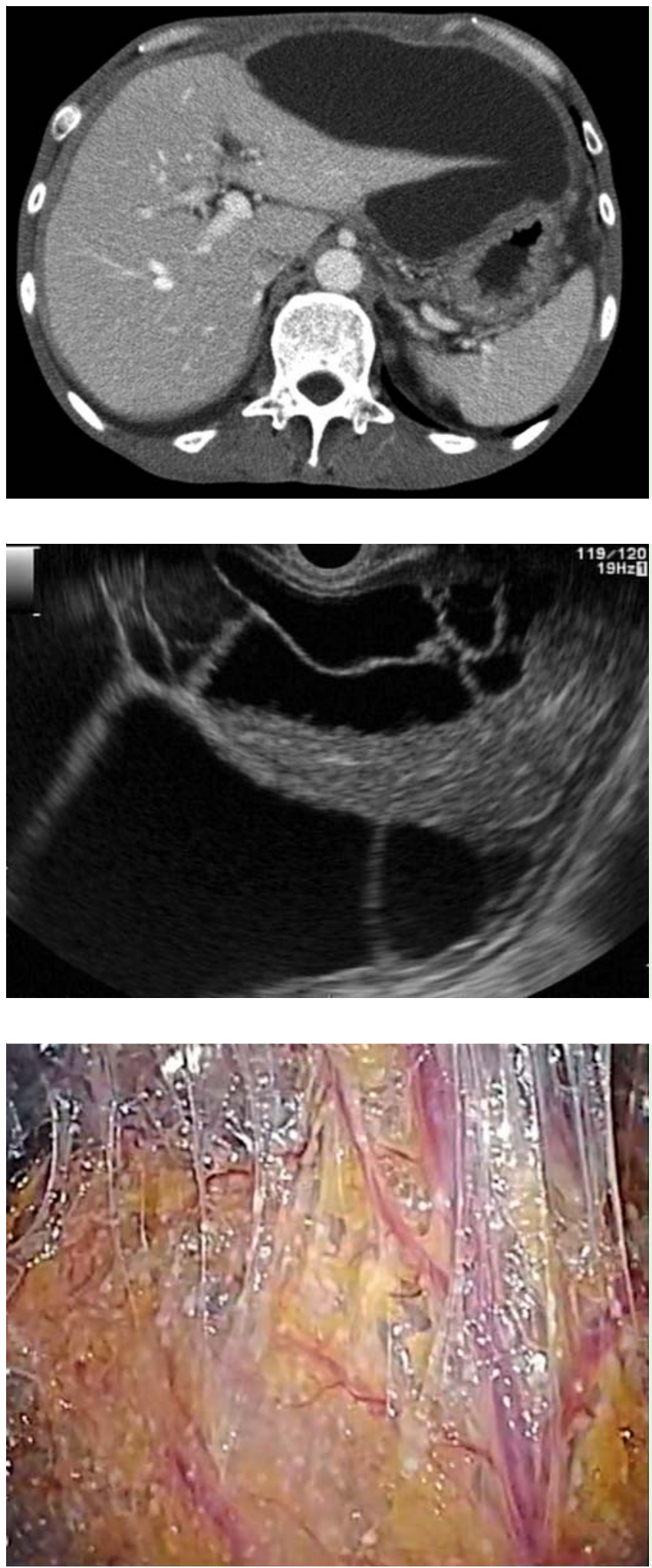

Fig. 1 Computed tomography showed a wall-enhanced cystic lesion in the lobe of the liver, with a maximum diameter of $200 \mathrm{~mm}$.

Fig. 3 Endoscopic ultrasonography showed the cyst with multilocular septa.

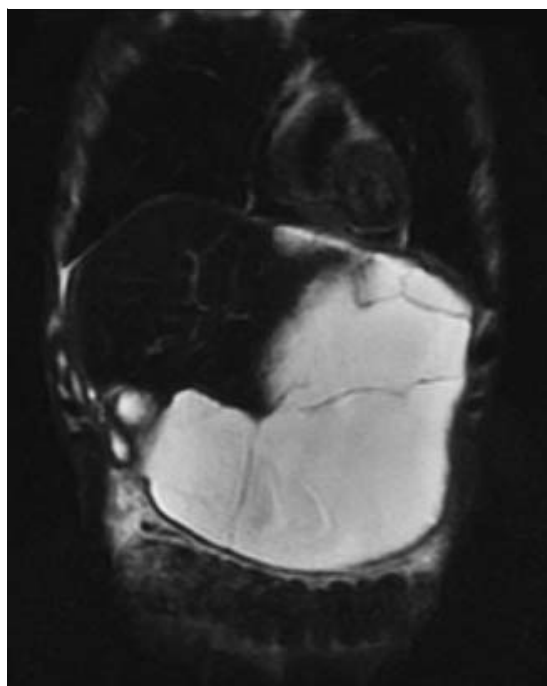

Fig. 2 T2-weight magnetic resonance imaging showed a hyperintense multiloculated cyst.

Liver involvement in tuberculosis infection is rare, and the radiological findings have previously been interpreted as multiple liver tumors, hemangioma, abscess, or cirrhosis [1 - 3]. Conversely, hepatic cyst is a common benign liver tumor that is usually easy to diagnose as a simple hepatic cyst. Despite advances in imaging techniques, differentiating complicated hepatic cysts from other tumors, including malignancies, sometimes remains challenging. We describe a rare case of a giant liver cyst caused by tuberculosis and masquerading as malignant peritonitis.

Fig.4 On laparotomy, many white nodules that were observed in the abdominal cavity were suspected to represent malignant peritonitis.
A 60-year-old man was referred to our hospital with a 3-week history of persistent postprandial nausea. Computed tomography showed a wall-enhanced cystic lesion in the left lobe of the liver, measuring $200 \mathrm{~mm}$ in maximum diameter ( Fig.1). Magnetic resonance imaging showed a multiloculated cyst with hyperintensity on T2-weighted imaging and decreased diffusion at the cystic wall on diffusion-weighted imaging (DWI) ( $\bullet$ Fig. 2). On endoscopic ultrasonography, the cyst had multilocular septa ( Fig.3). Because the patient remained symptomatic, and DWI suggested the possibility of malignancy, we decided that surgery should be performed.

Laparotomy revealed many white nodules in the abdominal cavity, suggesting malignant peritonitis ( Fig.4). However, these nodules and the cystic wall included gran- 


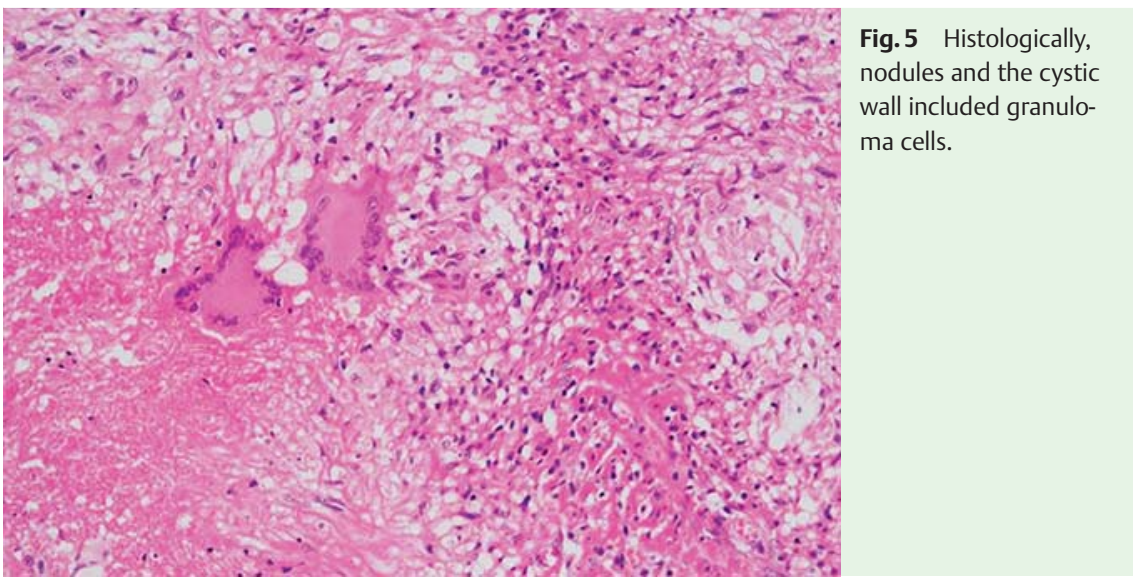

uloma cells as diagnosed by histology during intraoperative rapid diagnosis; these results suggested tuberculosis ( $\bullet$ Fig.5). Positive culture results were subsequently obtained for tubercle bacillus from ascites fluid and blood samples. The cyst was surgically removed, and the patient underwent antituberculosis treatment (isoniazid, rifampicin, pyridoxal phosphate hydrate) for 6 months postoperatively. This case may represent the first report of a giant complicated cyst caused by tubercular involvement of the liver. While various diagnostic imaging modalities have been developed, the possibility of giant liver cysts caused by tuberculosis should be considered in order to prevent misdiagnosis.
Endoscopy_UCTN_Code_CCL_1AG_2AB

Competing interests: None

Takeshi Ogura', Yoshitaka Kurisu², Daisuke Masuda ${ }^{1}$, Akira Imoto', Saori Onda', Rieko Kamiyama', Nobu Nishioka', Kazuhisa Uchiyama ${ }^{3}$, Kazuhide Higuchi ${ }^{1}$

1 2nd Department of Internal Medicine, Osaka Medical College, Osaka, Japan

${ }^{2}$ Department of Pathology, Osaka Medical College, Osaka, Japan

${ }^{3}$ Department of General and Gastroenterological Surgery, Osaka Medical College, Osaka, Japan

\section{References}

1 Singh D, Singh S, Raut SB et al. Isolated hepatic tuberculosis: a case report. Pediatr Surg Int 2004; 20: 727-728

2 Bangroo AK, Malhotra AS. Isolated hepatic tuberculosis. J Indian Assoc Pediatr Surg 2005; 10: 105-107

3 Tai WC, Kuo CM, Lee $\mathrm{CH}$ et al. Liver tuberculosis in Southem Taiwan: 15-years clinical experience. J Intern Med Taiwan 2008; 19: 410-417

\section{Bibliography}

DOI http://dx.doi.org/

10.1055/s-0034-1365149

Endoscopy 2014; 46: E191-E192

(c) Georg Thieme Verlag KG

Stuttgart · New York

ISSN 0013-726X

\section{Corresponding author \\ Takeshi Ogura, MD, PhD}

2nd Department of Internal Medicine

Osaka Medical College

1-1 Daigakuchou

Takatsukishi, Osaka 464-8681

Japan

Fax: +81-527635233

oguratakeshi0411@yahoo.co.jp 